\title{
Highly Scalable Amplified Hybrid TDM/DWDM Array Architecture for Interferometric Fiber-Optic Sensor Systems
}

\author{
Yi Liao, Ed Austin, Philip J. Nash, Stuart A. Kingsley, Senior Member, IEEE \\ and David J. Richardson, Senior Member, IEEE
}

\begin{abstract}
We present a distributed amplified hybrid dense wavelength division multiplexing (DWDM) and time division multiplexing (TDM) array architecture for large scale interferometric fiber-optic sensor array systems. This architecture employs a distributed Erbium doped fiber amplifier (EDFA) scheme to decrease the distribution loss among multiplexed wavelengths, and employs TDM at each wavelength to increase the total number of sensors that can be supported. The first experimental demonstration of this system is reported including results which show the potential for multiplexing and interrogating up to $\mathbf{4 0 9 6}$ sensors using a single telemetry fiber pair with good system performance. The number of interrogation sensors could be further increased by increasing the number of wavelength channels. These architectures would be of great importance in the application of systems requiring very large number of sensors with limited telemetry cabling.
\end{abstract}

Index Terms-Amplified array, large scale, distributed amplification, interferometric fiber optics sensors, Erbium doped fiber amplifiers, hybrid multiplexing, phase resolution, noise aliasing

\section{INTRODUCTION}

Fiber-optic sensors provide many advantages over conventional electro-ceramic-based devices, including their immunity to electromagnetic interference, high sensitivity, simplicity, smaller cross-section, potential lower cost, ease of multiplexing and in particular reliability in underwater applications. Interferometric fiber optic sensors have been researched for nearly four decades for a range of practical applications, including in particular military sonar and seismic surveying [1] which have been the main drivers behind the development of optical fiber sensor-based hydrophone arrays.

Interferometric fiber optic sensor arrays are usually based on measuring the phase modulation of light travelling in an optical fiber due to the strains developed on the fiber by a measurand. Even with the ultra-high sensitivities now achieved, single channel applications are only appropriate in a few instances, mostly for cost reasons. Fortunately, fiber-optic sensors lend themselves to multiplexing, which allows the cost of the lasers

${ }^{1}$ Manuscript received September, 2012.

Yi Liao and David J. Richardson are with the Optoelectronics Research Centre, University of Southampton, UK (phone: 44-23-80595343. e-mail: yil@orc.soton.ac.uk, djr@orc.soton.ac.uk).

Ed Austin, Philip J. Nash and Stuart A. Kingsley are with TGS, Surrey KT6 6AP, UK (e-mail: $\quad$ Ed.Austin@tgs.com, Phil.Nash@tgs.com, skingsley@fiberdyneoptoelectronics.com). and other expensive components to be divided between many channels. Many multiplexing schemes have been proposed and investigated based on techniques including time, frequency, coherence, and wavelength multiplexing, and combinations thereof [2-6]. In each instance splitting/recombination loss ultimately limits the scalability of the approach, with the number of fibers required for telemetry a further critical factor that significantly impacts the overall system cost and practicality. Erbium doped fiber amplifiers (EDFAs) are widely used in interferometric fiber sensing systems, frequently to compensate for splitting loss as well as inherent fiber transmission loss. Pre-amplifiers, post-amplifiers and in-line amplifiers as single amplifiers are all often used [7, 8]. The largest interferometric fiber-optic sensor array reported to date consisted of a time- and wavelength-division multiplexed architecture combining up to 256 sensors onto a single fiber pair [9].

Among different interferometric sensor multiplexing techniques, time division multiplexing has been shown to have low crosstalk and high sensitivity [5, 10]. In the TDM scheme, sensors are sequentially addressed using a pulsed input signal such that the time of flight of optical pulses in the multiplexed array allows individual sensor signals to be distinguished. In a TDM scheme, the maximum number of sensors per fiber pair is limited by the distribution losses. Nevertheless, compensation of splitting loss as well as the fiber loss can be achieved by incorporating EDFAs in the array. For example, high-gain preand post-amplifiers were used in a 64-element interferometric sensor by researchers from the Naval Research Laboratory (NRL) [8]. They work well, but the increased number of sensors requires higher input signal powers, and nonlinear effects on the buses ultimately limit the maximum number of sensors to 96.

A better solution has been developed in which an amplifier is placed in front of each distribution coupler on both buses in the TDM scheme [3, 11-13]. A system was demonstrated that consisted of a ten-rung ladder structure using multiple low-gain amplifiers to overcome the branching loss in each ladder rung. This method allows 200 sensors to be addressed by only one fiber pair with a minimum detectable signal of $5.7 \mu \mathrm{rad} / \sqrt{ } \mathrm{Hz}$ [13]. However, it is increasingly difficult to support more sensors beyond a total number of about 300 in a TDM scheme due to nonlinear effects, and the optical duty cycle or interrogation rate of each sensor [5]. 


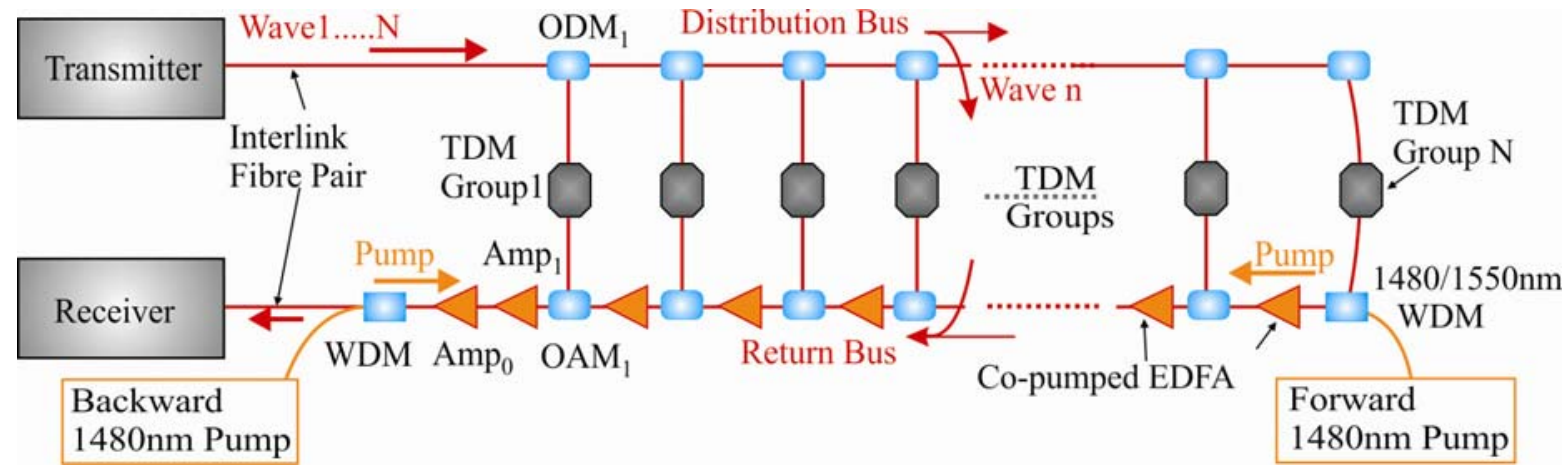

Fig.1 Proposed amplified TDM /DWDM array topology, WDM: 1480/1550nm Wavelength Division Multiplexing, ODM: optical drop multiplexer, OAM: optical add multiplexer, TDM group: time division multiplexed sensor group (all sensors driven by one wavelength), EDFA: Erbium doped fiber amplifier.

Compared to the TDM architecture, the combination of DWDM with a TDM architecture is significantly more efficient, both in terms of number of channels per fiber and number of channels per laser [9, 13]. DWDM allows many TDM signals at different wavelengths to be combined on a single fiber simultaneously, such that the multiplexing factor is now given by the product of the number of TDM sensors (typically $\sim 30$ ) and the number of wavelengths. This architecture has demonstrated high performance from sensors in an optically efficient arrangement with low component numbers, and can theoretically be extended to interrogate at least 192 [10] 256 [9] sensors through two fibers while achieving a low-phase resolution limited by the optical amplifier noise. However, the optical power levels received for signals significantly decreases with increasing array size.

This paper extends this technique to a novel high performance fiber-optic sensor array scheme using a distributed EDFA and hybrid TDM/DWDM. This novel architecture is considered be the best approach to support high resolution, high sensor count interferometric sensor arrays (we show viability of up to 4096 sensors in this work). Moreover it uses only commercially available components through one pair of telemetry fibers. In section II, we describe the operational principle of the amplified TDM/DWDM array architecture. Section III demonstrates the experimental arrangement and power budget. Section IV presents the optical performance and the phase noise performance of the lowest resolution sensor as a function of signal power. Section V presents a system model to determine the sensor performance in such systems and discusses the potential sensor number which the array architecture can be scaled up to. Section VI summarizes the conclusions.

\section{PRINCIPLE OF OPERATION}

The principle of the array topology proposed here is based on loss compensation of the distribution bus using a distributed multi-section EDFA in a hybrid TDM/DWDM architecture. The array is addressed via one interlink fiber pair, as illustrated in Fig. 1. The optical pulses from the wavelength multiplexed sources are coupled into the distribution bus. At the first optical drop multiplexer (ODM), the signal at wavelength $\lambda_{1}$ is coupled into the first TDM group (all sensors driven by one wavelength), passes through the constituent sub-array of TDM sensors, and is then coupled onto the return bus through the optical add multiplexer (OAM), which delivers it to the detector. The remaining wavelengths of the input interrogation signal continue along the distribution bus to the subsequent ODMs, successively feeding into each TDM group, before being multiplexed onto the return bus. This architecture performs the 'add' and 'drop' functions by separate devices and thus provides the advantage that if the telemetry fiber is severed within the array, then only the signals from the TDM groups corresponding to ODM/OAMs positioned after the break are lost.

To balance the powers among different channels, EDFA segments are distributed along the return bus to compensate the insertion loss of the OAM/ODMs, thus the optical signals from all the TDM sensor groups now experience approximately the same net optical gain. Amplifiers are applied only on the return bus, because the travelling optical power on the distribution bus is beyond the EDFA's small-signal regime, i.e. where the gain is independent of the input signal power. Another secondary reason is that no additional pumps and multiplexers are required on the distribution bus, which simplifies the system and lowers the cost. Since only wavelengths out of the add channel bandwidth $(\sim 0.7 \mathrm{~nm})$ are passed through the express channel of the OAMs on the return bus and there exists a measured stop band attenuation of $\sim 20 \mathrm{~dB}$ at the OAM express port, this architecture also provides a partial rejection effect on the accumulated in-band amplified spontaneous emission (ASE).

The gain of each amplifying section is set to exactly compensate for the subsequent losses for each sensor group. The amplifiers are all co-pumped remotely through the existing return bus fiber by $1480 \mathrm{~nm}$ pump lasers located at the front and/or back ends of the array. Since each amplifier must compensate for only a small insertion loss (except for $\mathrm{Amp}_{0}$ ), they provide a low gain and require just a small pump power.

The input pump power is selected to be much larger than the first amplifier's saturation pump power, and the couplers are optical drop/add multiplexers so that the pump never couples out of the buses. Therefore this amplifier absorbs only a small fraction of the pump power, so that the large remaining power is transmitted to the downstream amplifiers, which also operate in 


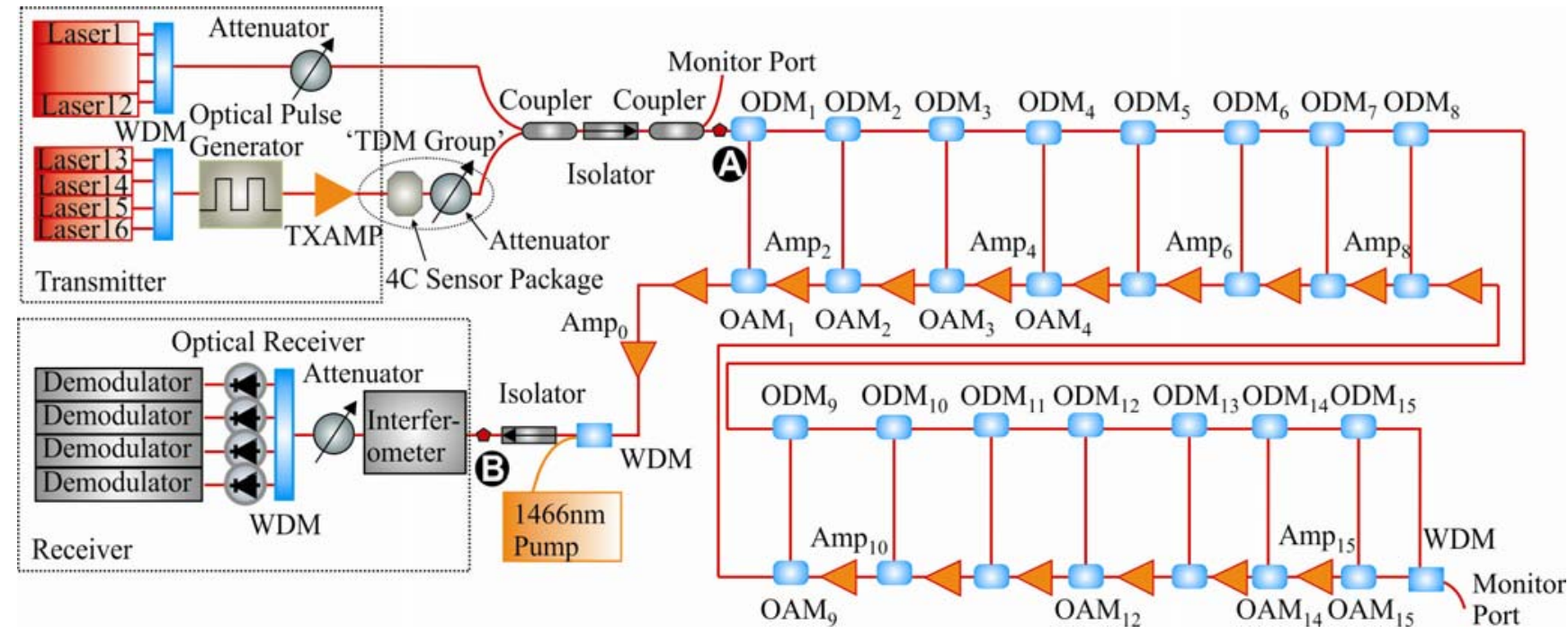

Fig.2 Experimental setup of the 16 wavelength architecture for phase noise floor interrogation from four narrow linewidth fiber lasers (laser 13 to laser 16) and 12 representative DFB lasers (laser 1 to laser 12), WDM: Wavelength Division Multiplexing, TXAMP: Amplifier in the transmitter.

a pump saturation regime. The 1480 pump wavelength is chosen due to the low transmission loss. It is thus possible to pump tens of widely distributed low-gain amplifiers with a modest pump power. Since each amplifier is highly inverted and provides low gain, the noise is low.

This array architecture provides dramatic advantages for large scale arrays. The topology uses TDM within an amplified DWDM architecture for the first time, such that the number of multiplexed sensors is now given by the product of the number of TDM sensors and the number of wavelengths, which is increased by several times compared to the number multiplexed in amplified TDM arrays with the same interrogation repetition rate [3]. The interrogation repetition rate determines the bandwidth available for the phase-modulated signal to occupy, and it is dependent on the number of sensors and the length of fiber per sensor. The array allows the interrogation of thousands of multiplexed sensors utilizing only one single telemetry fiber pair, which significantly decreases the array complexity, cost, weight, and provides for ready expansion. The insertion loss of the array is compensated by the distributed amplification, which leaves a higher power budget for the remote transmission of the signal along the cable, and limits the chance of nonlinear effects within the fiber bus. These benefits are of prime importance in many applications.

\section{EXPERIMENT ARRANGEMENT}

\section{A. Experiment arrangement}

Although a larger number of wavelengths can be multiplexed, due to cost, practicality and equipment availability, we use 16 wavelengths in our proof-of-principle experimental configuration, as shown in Fig.2. This sensor system comprises three principal components: a transmitter, the amplified array architecture and a receiver section. The transmitter consists of four narrow linewidth $(\sim 10 \mathrm{kHz})$ fiber lasers for interrogation, and a further twelve DFB lasers (from a DWDM telecom source bank) to provide suitable representative signals to assess the optical amplification performance of the array. (The linewidth of the DFB lasers was too broad to allow them to be used for sensor interrogation.) The four narrow linewidth sources were multiplexed, pulsed, frequency-shifted, and amplified, then launched into a "TDM group". The output signal was then combined with the twelve $\mathrm{CW}$ sources and then launched into the amplified array architecture. The maximum launch power into the array was $+22 \mathrm{dBm}$ per wavelength, limited by nonlinear effects.

The experimental arrangement was constructed with only one "TDM group" representing all of the sensors in the array. This was located before the array so that each wavelength suffered the loss of this device. This should provide similar optical performance in terms of loss to a fully-loaded system in which a TDM group is included between each ODM/OAM pair and is obviously far more convenient from a practical perspective. The "TDM group" itself comprised a lab 4C sensor package along with a tunable attenuator to simulate a prescribed number of additional "missing sensors" as described below. The lab $4 \mathrm{C}$ sensor package is made of a cluster of three orthogonally mounted accelerometers and a hydrophone as described in [9]. Later we will show the measured phase noise floor of the sensors in the $4 \mathrm{C}$ sensor package for characterization of the system performance.

The amplified array was comprised of 15 pairs of ODM/OAMs along two fiber buses to support the 16 wavelengths. The insertion losses of the ODM/OAMs were measured to determine the lengths of the Er-doped fiber required in each amplifier to compensate their loss. An amplifier was located after each ODM/OAM pair. In our experiments all amplifiers were co-pumped by a $1466 \mathrm{~nm}$ pump source (due to lab limitations) coupled into the return bus with a maximum output pump power of $400 \mathrm{~mW}$. The lengths of 
amplifier segments used throughout the network were determined by detailed numerical simulations of the system, supplemented by detailed amplifier measurements which allowed us to determine the salient physical properties of the erbium doped fiber used.

The return signals from the array were referenced at an interferometer for subtraction of system generated noise, and attenuated before the demultiplexer to achieve a peak optical power of $-27 \mathrm{dBm}$ per wavelength at the optical receiver as required to obtain the desired shot noise limited performance. The demultiplexer was used to drop the signal bearing channels. The output of the demultiplexer was then detected and demodulated to extract the phase information.

\section{B. Power budget}

The system performance in an amplified array is ultimately limited by the ASE noise from the amplifiers. The injected average signal power into the EDFA segments determines the amplifier performance. The insertion loss (IL) of one sensor $l_{s}$ was assumed to be $5 \mathrm{~dB}$, increasing by $6 \mathrm{~dB}$ for every doubling of the number of sensors in the TDM group, as is consistent with current sensor technology. The value of $6 \mathrm{~dB}$ accounts for the insertion loss of the return of light across an additional 1:1 coupler. Assuming the returned optical signal from the TDM group is fully occupied in the time domain by interleaving the pulse returns from different sensors in the group, i.e., the number of the sensors is the reciprocal of the duty cycle of the input pulse, then the returned average optical power equals the pulse peak power. When an optical peak power of $+22 \mathrm{dBm}$ per wavelength is launched into a fully-loaded system in which a TDM group is included between each ODM/OAM pair, the optical power to the $A_{m p}$ can be found. In the proof-of-concept experiment, we manually set the number of sensors in each TDM group by adjusting the IL in the "TDM group" located before the amplified array for simplicity, thus the power we monitored at point $\mathrm{A}$ equates to the injected power to $\mathrm{Amp}_{0}$ in a practical system.

TABLE 1 POWER BUDGET PER CHANNEL WITH +22 DBM LAUNCHED POWER INTO THE ARRAY

\begin{tabular}{ccc|c}
\hline $\begin{array}{c}\text { Number of } \\
\text { Sensors in each } \\
\text { TDM group }\end{array}$ & $\begin{array}{c}\text { IL } \\
(d B)\end{array}$ & $\begin{array}{c}\text { Signal power } \\
\text { to Amp } \\
(d B m)\end{array}$ & $\begin{array}{c}\text { Signal power } \\
\text { at A in the setup } \\
(d B m)\end{array}$ \\
\hline 1 & 5 & 17 & 17 \\
4 & 17 & 5 & 5 \\
16 & 29 & -7 & -7 \\
64 & 41 & -19 & -19 \\
128 & 47 & -25 & -25 \\
256 & 53 & -31 & -31 \\
512 & 59 & -43 & -43 \\
\hline
\end{tabular}

\section{EXPERIMENT RESULTS}

We first characterized the narrow linewidth fiber lasers (ROCK laser modules from NP Photonics Inc.) used for interrogation, since their noise performance determines the best resolution that can be achieved in such interferometric sensor systems. The laser frequency noise can be included by assuming that the interferometer time imbalance is the input pulse period and plotting a best fit line through the phase noise spectrum of the laser, thus the phase noise amplitude, in $\mathrm{rad} / \sqrt{\mathrm{Hz}}$, due to the laser frequency noise is given by $\delta \phi(f)$. The measured phase noise spectrum from a delayed Mach-Zehnder (MZ) interferometer normalized to one meter of optical path difference (OPD) [14] is shown in Fig. 3. Relative intensity noise (RIN) from the laser is equivalent to an amplitude modulation of the optical signal, causing the RIN spectrum to appear as amplitude modulation sidebands around the carrier. The equivalent noise contribution due to the RIN is thus expressed as $\operatorname{RIN}(f)$. The measured RIN spectrum is also characterized in Fig. 3 [14]. The laser RIN noise spectrum is dominated by a peak at the relaxation frequency of the laser around $1 \mathrm{MHz}$ and is shot-noise limited otherwise. This relaxation frequency also leads to a peak in the frequency noise spectrum.

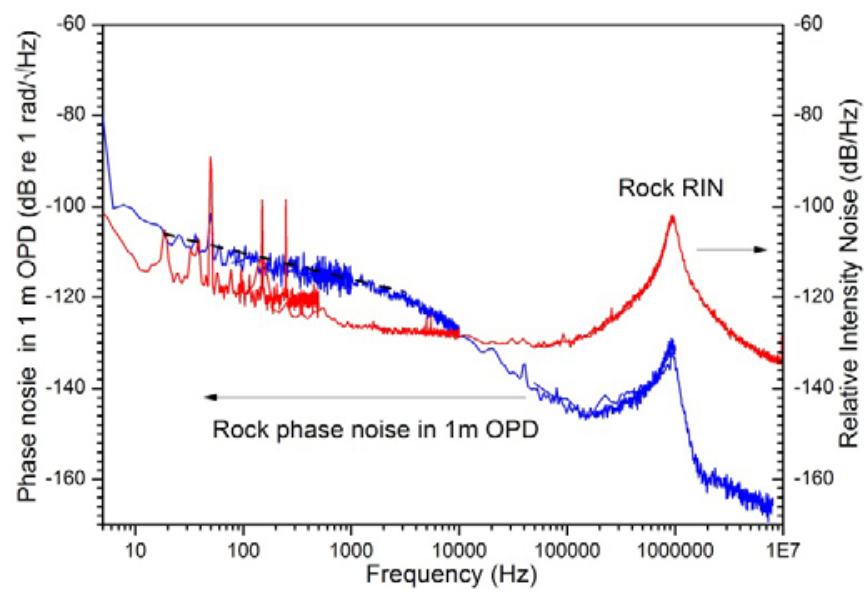

Fig. 3 Noise characterization of the Rock laser used in the proof-of-concept experimental setup, including phase noise spectrum in one-meter OPD and the relative intensity noise spectrum.

When determining the amplification performance of the array with a distributed EDFA, we assume that all the amplifiers have the same gain $G_{i}$, equal to the transmission loss between amplifier stages along both of the return bus $\left(l_{p} l_{x} l_{p} l_{x}\right)$. The gain of the $\mathrm{Amp}_{0}$ is $G_{0}$. The stimulated emission in each amplifier is accompanied by spontaneous emission, and it will be amplified by following amplifier stages. Thus the accumulated ASE noise power at the output of $\mathrm{Amp}_{0}$ after multiple amplifications is given by

$$
P_{A S E}=2 n_{s p} h v B_{o}\left\{\frac{G_{i}-1}{G_{i}}\left[\frac{1-\left(\mathrm{l}_{\mathrm{p}} \mathrm{l}_{\mathrm{x}} \mathrm{G}_{i}\right)^{N-1}}{1-1_{\mathrm{p}} \mathrm{l}_{\mathrm{x}} \mathrm{G}_{i}}\right]+\frac{G_{0}-1}{G_{0}}\right\} G_{0},
$$

where

$B_{o}$ is the optical bandwidth,

$n_{s p}$ is the spontaneous emission factor of the amplifiers,

$h$ is the Plank constant,

$v$ is the optical frequency,

$G_{0}$ is the gain in $\mathrm{Amp}_{0}$,

$\mathrm{N}$ is the number of amplifier stages.

It should be mentioned here that OAMs can reject the ASE noise power accumulated from earlier amplifier stages at the 
multiplexing channel, thus the ASE noise at the first channel is several $\mathrm{dB}$ lower than the worst (last) one. If the input signal

launched to the array is $P_{i n}$, with $G_{0} \gg 1$, thus $G_{0} /\left(G_{0}-1\right) \approx 1$,

we get the worst optical signal to noise ratio (OSNR) at the last channel

$$
O S N R=\frac{P_{i n}\left(l_{p} l_{x}\right)^{2(N-1)} G_{i}^{N-1}\left(l_{a d}\right)^{2}\left(l_{s} / M^{2}\right)}{2 n_{s p} h v B_{o}\left\{\frac{G_{i}-1}{G_{i}}\left[\frac{1-\left(\mathrm{l}_{\mathrm{p}} \mathrm{l}_{\mathrm{x}} \mathrm{G}_{i}\right)^{N-1}}{1-\mathrm{l}_{\mathrm{p}} \mathrm{l}_{\mathrm{x}} \mathrm{G}_{i}}\right]+1\right\}},
$$

in which $\mathrm{M}$ is the number of sensors in the TDM group and $l_{s}$ denotes the IL of a single senor. The OSNR worsens by the first term in the square brackets of the division in (2) relative to the OSNR in a passive array. This term accounts for the accumulated ASE noise from earlier stages. The optical spectra before and after the amplified array were characterized with a launched power at $\mathrm{A}$ of $-31 \mathrm{dBm}$, as shown in Fig.4. The spectrum resolution used to obtain the power spectral density (PSD) is $0.1 \mathrm{~nm}$. It can be seen from the spectra that the OSNR for each channel is decreased by the ASE noise added by the distributed EDFA. The output optical spectrum around the signal wavelengths dipped below the ASE power level because of the filter effect on the express port of the OAMs along the return bus as stated previously. Thus the OSNR at $\mathrm{Ch} 1$ is $5 \mathrm{~dB}$ better than the worst one due to this 'partial rejection effect' from the OAMs.

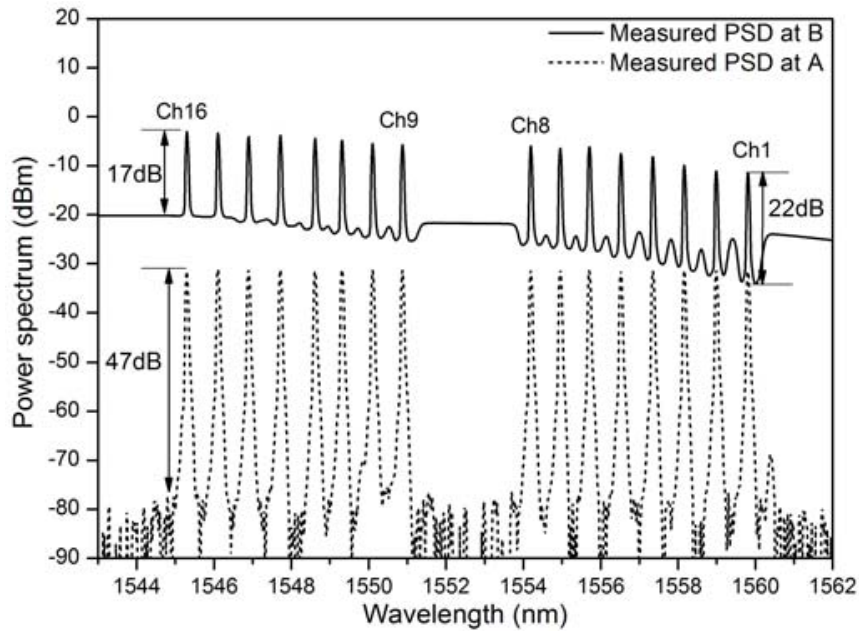

Fig.4 Optical power spectral density measured at point $\mathrm{A}$ and $\mathrm{B}$ in the proof-of-concept experimental setup, with the TDM group insertion loss of 53 $\mathrm{dB}$ equivalent to 256 sensors per wavelength. The OSNR at the last (also the worst) channel decreases from $47 \mathrm{~dB}$ to $17 \mathrm{~dB}$ due to the accumulated ASE noise.

The expression also shows that the channel OSNR increases with the signal power $\mathrm{P}_{\text {in }}$ along the return bus. We get the predicted and measured OSNR for the last channel at point B as a function of the launched power to the array at point $\mathrm{A}$ in the proof-of-concept experimental arrangement, as illustrated in Fig. 5, with measured $l_{p} l_{x}=0.25 \mathrm{~dB}, G_{i}=0.9 \mathrm{~dB}$, and assumed $\mathrm{n}_{\mathrm{sp}}=1$ due to the practical low noise amplifier performance. This figure illustrates the linear relationship between the channel OSNR and the launched signal power level. The predicted and measured OSNR are in good agreement. The values along the top axis show the equivalent number of sensors in each TDM group to achieve those OSNRs. Even with 64 sensors per wavelength, the OSNR at the worst channel is still better than $29 \mathrm{~dB}$.

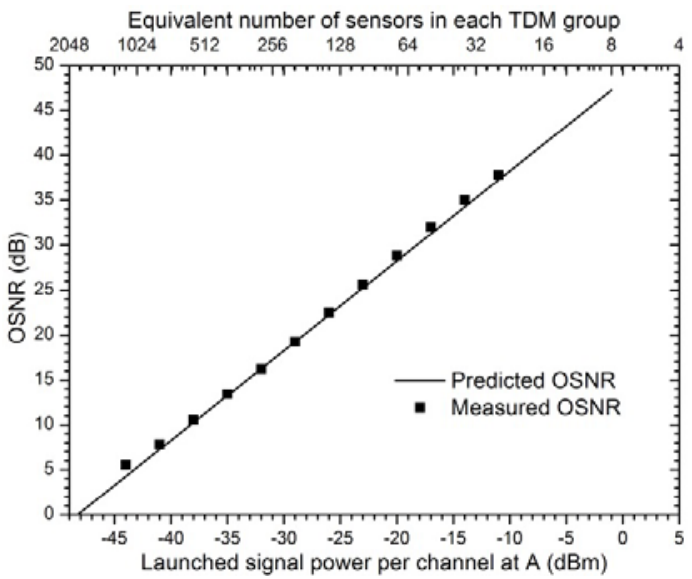

Fig. 5 Measured and predicted OSNR as a function of the optical signal power per channel at point A. The values along the top axis show the equivalent number of sensors in each TDM group to achieve those OSNRs with a launched power of $+22 \mathrm{dBm}$ into a fully-loaded system.

To illustrate system performance, the phase noise spectrum of one sensor in the 'TDM group' was tested under a wide range ( 23 to $59 \mathrm{~dB}$ ) of TDM group insertion loss. Fig. 6 shows the demodulated phase spectrum range from $100 \mathrm{~Hz}$ to $2 \mathrm{kHz}$ interrogated at the last channel $(1545.32 \mathrm{~nm})$ with a TDM group IL of $35 \mathrm{~dB}$. The IL of the 'TDM group' for the illustrated spectrum in the figure is close to the loss of a TDM group with 32 sensors addressed by a single wavelength. This equates to a total number of 512 sensors in the system. The system phase noise floor shows a flat spectrum from 100 to $2000 \mathrm{~Hz}$, achieving a value around $-88 \mathrm{~dB}$ re $1 \mathrm{rad} / \sqrt{\mathrm{Hz}}$, thus we define the demodulated phase resolution in the sensor system as the mean value of the system phase noise floor from 300 to $800 \mathrm{~Hz}$, and the best system resolution is calibrated to be $-90 \mathrm{~dB}$ re 1 $\mathrm{rad} / \sqrt{\mathrm{Hz}}$ in a passive system limited only by the laser noise. (Below $100 \mathrm{~Hz}$, the noise floor is limited by environmental noise.)

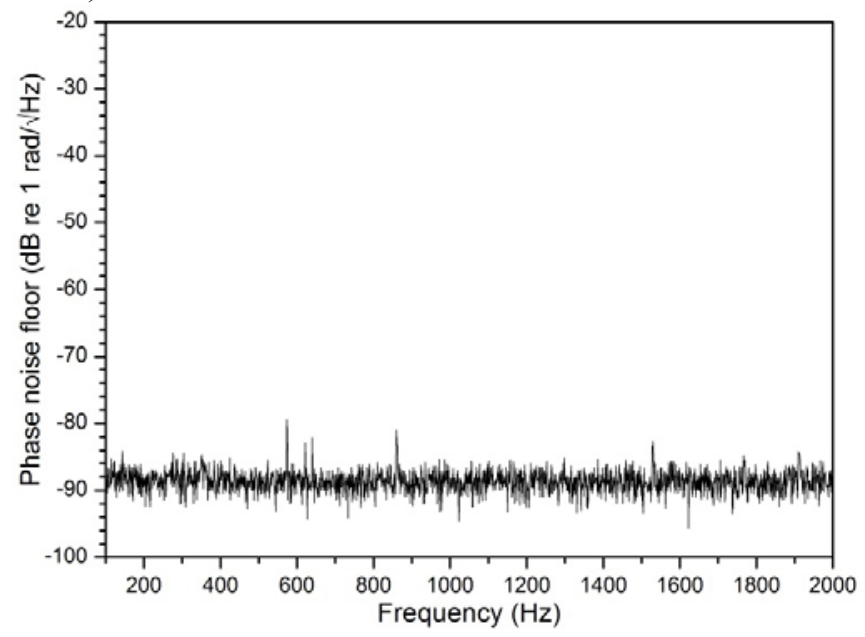


Fig. 6 Demodulated phase spectrum from a sensor in the 'TDM group' with 16 wavelengths in the array for a launched power at A of $-13 \mathrm{dBm}$, with a TDM group insertion loss equivalent to 32 sensors per wavelength.

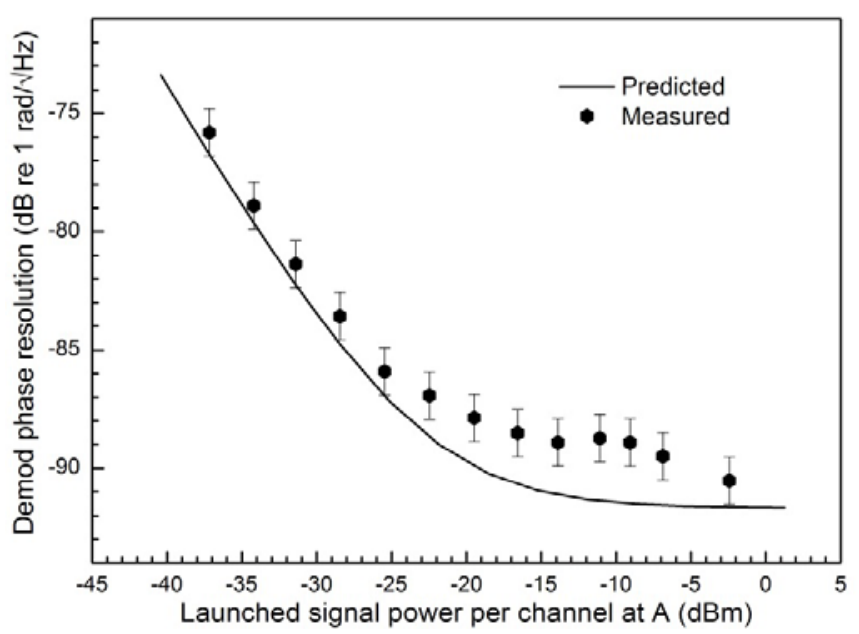

Fig. 7 Measured and predicted demodulated phase resolution peak value as a function of the launched signal power, with IL of the 'TDM group' range from $23 \mathrm{~dB}$ to $59 \mathrm{~dB}$, equivalent to $8 \times 16$ to $512 \times 16$ sensors in the array.

Fig. 7 demonstrates the measured and predicted demodulated phase resolution as a function of the launched optical power at point $\mathrm{A}$ in the proof-of-principle experimental setup (Fig. 2). The method to predict the phase resolution in a fiber optic interferometric sensor system will be presented in detail in a companion paper [15]. This phase noise model covers all the major noise sources which affect the system resolution, including the shot noise, ASE noise, receiver noise, RF (radio frequency) oscillator noise and nonlinear effect noise sources, in which the shot noise dominates, but the ASE induced noise starts to dominate when the signal OSNR deteriorates. It can be seen from the curve that the demodulated phase noise starts to increase from $-88 \mathrm{~dB}$ re $1 \mathrm{rad} / \sqrt{\mathrm{Hz}}$, when the launched power is smaller than $-19 \mathrm{dBm}$. This equates to a total of $64 \times 16$ sensors in the system. This can be explained as follows. Due to the amplification employed in the array, the signal-amplified spontaneous emission (ASE) beat noise contributes to the system noise floor. In the small-signal regime, due to the high population inversion a low noise figure is obtained and the generated ASE noise can be ignored. However, as the IL of the
TDM group is increased, a higher gain is required and a lower input signal is injected at the EDFA input. ASE builds up rapidly and starts to help saturate the EDFA giving rise to signal-ASE beat noise which ultimately becomes the dominant noise source and increases the system noise floor as the number of sensors per wavelength is increased. Our results show that the experimental arrangement can address up to $64 \times 16$ sensors with a phase resolution limited only by the noise from the fiber lasers.

\section{SYSTEM MODEL: PHASE RESOLUTION AND MAXIMUM SENSOR NUMBER}

The sensor system's interferometric phase resolution is determined by the signal noise power ratio at the receiver [16] and other phase noise contributions from the laser and nonlinear effects. The induced peak phase noise is given by

$$
\delta \phi=\sqrt{\frac{2\left\langle i_{n}^{2}\right\rangle}{\left(R P_{s}\right)^{2}}+2 \delta^{2} \phi_{n}} .
$$

$\overline{i_{n}^{2}}$ and $\delta^{2} \phi_{n}$ are the intensity noise power and the phase noise at the receiver. $P_{s}$ is the optical power at the receiver. Typically in interferometric sensor systems, the phase resolution is limited by the signal shot noise, receiver noise, RF oscillator noise, laser intensity noise and frequency noise. However, in practice, the high frequency noise will alias to the demodulation bandwidth.

In systems with limited detection bandwidth of $B_{e}$, the broadband noise is aliased at a signal frequency of $f_{m}$. The effect of aliasing can be assessed by the 'Demod phase resolution' [15], with its peak value given by

$$
\delta \phi_{\text {Demod }}=2 \sqrt{\sum_{k}\left[2 \sum_{q=0}^{\left(B_{e}-f_{e}-f_{m}\right) / F_{s}} \delta \varphi_{k}^{2}\left(f_{e}+f_{m}+q F_{s}\right)\right]} .
$$

in which $F_{s}$ represents the pulse repetition rate, $k$ stands for the subscript of different noise sources, $f_{e}$ denotes the effective starting frequency to be aliased at various signals for different noise sources, and $q$ is an aliasing coefficient.

In our proof-of-concept experimental setup, the system Demod phase resolution peak value was characterized in Fig. 7.

\begin{tabular}{|c|c|c|c|c|c|c|c|}
\hline $\begin{array}{c}I L \\
(d B)\end{array}$ & $\begin{array}{l}\text { Measured Demod } \\
\text { phase resolution } \\
(\mathrm{dB} \text { re } 1 \mathrm{rad} / \sqrt{ } \mathrm{Hz})\end{array}$ & $\begin{array}{c}\text { Equivalent number of } \\
\text { sensors per wavelength }\end{array}$ & $\begin{array}{l}\text { Total number } \\
\text { of sensors }\end{array}$ & $\begin{array}{c}\text { Phase resolution } \\
(\mu \mathrm{rad} / \sqrt{ } H z)\end{array}$ & $\begin{array}{c}\text { Pulse } \\
\text { width (ns) }\end{array}$ & $\begin{array}{l}\text { Sampling } \\
\text { rate } \\
(\mathrm{Hz})\end{array}$ & $\begin{array}{c}\text { Predicted Demod } \\
\text { phase resolution } \\
(\mathrm{dB} \text { re } 1 \mathrm{rad} / \sqrt{\mathrm{Hz}})\end{array}$ \\
\hline 5 & -92 & 1 & 16 & 0.7 & & $200 \mathrm{k}$ & -91 \\
\hline 17 & -90 & 4 & 64 & 0.9 & 100 & $200 \mathrm{k}$ & -90 \\
\hline 29 & -90 & 16 & 256 & 0.9 & 25 & $200 \mathrm{k}$ & -90 \\
\hline 41 & -88 & 64 & 1024 & 1.1 & 25 & $200 \mathrm{k}$ & -88 \\
\hline 47 & -85 & 128 & 2048 & 1.6 & 25 & $100 \mathrm{k}$ & -82 \\
\hline 53 & -83 & 256 & 4096 & 2 & 25 & $50 \mathrm{k}$ & -77 \\
\hline 59 & -76 & 512 & 8192 & 4.6 & 25 & $25 \mathrm{k}$ & -69 \\
\hline
\end{tabular}

TABLE 2 POWER BUDGET PER CHANNEL WITH +22 DBM LAUNCHED POWER INTO THE ARRAY 
The first two columns in Table 2 demonstrate the relationship between the IL of the 'TDM group' and the measured Demod phase noise resolution. The middle columns in the table illustrate the predicted phase resolution (in $\mu \mathrm{rad} / \sqrt{\mathrm{Hz}}$ ) as a function of the total number of sensors in the 16 wavelength system. The phase resolution remains the same level when sensor numbers increase from one to 64 , with a phase resolution better than $1 \mu \mathrm{rad} / \sqrt{ } \mathrm{Hz}$, only limited by the laser frequency noise, similar to the performance of a passive array. They also show that the system can support up to 8192 sensors with a phase resolution less than $5 \mu \mathrm{rad} / \sqrt{\mathrm{Hz}}$, which is far better than the reported $\sim 100 \mu \mathrm{rad} / \sqrt{\mathrm{Hz}}$ phase resolution in a passive TDM/DWDM array with 384 sensors [2].

TDM architectures inherently sample each sensor at the interrogation repetition rate. It is to be appreciated that increasing the number of sensors per wavelength means decreasing the duty cycle in the time domain. For a limited pulse-width, the phase noise observed on each sensor increases with an increasing number of sensors in the TDM group, because the bandwidth available for each sensor decreases. Bandwidth limitations and added aliased high frequency noise in the TDM architecture ultimately limit the highest level of multiplexing possible, even with a suitably adjusted sampling rate, as illustrated in the middle columns in Table 2.

The table demonstrates the relationship between the number of sensors and the predicted phase resolution with a practical sampling rate and pulse-width in a practical system with existing demodulation technology. Nevertheless, these results show that the experimental arrangement can address up to $64 \times$ 16 sensors with a phase resolution limited only by the noise from the fiber lasers, and can support up to $256 \times 16$ with a perfectly acceptable phase resolution for many applications of $-77 \mathrm{~dB}$ re $1 \mathrm{rad} / \sqrt{ } \mathrm{Hz}$, even with an adjusted sampling rate. In this proof-of-principle experimental arrangement, the number of wavelengths has been chosen to be 16 . This can be further increased by adding more pump sources into the array.

\section{CONCLUSION}

In conclusion, we have demonstrated what we believe to be the first fiber optic sensor array scheme in a distributed amplified array based on a hybrid TDM and DWDM architecture. Our experimental results show that the array is able to support a total number of 1024 sensors along one interlink fiber pair, allowing a phase resolution of around -88 $\mathrm{dB}$ re $1 \mathrm{rad} / \sqrt{ } \mathrm{Hz}$ limited only by laser noise. Further, we show that the current 16-wavelength array could theoretically be expanded to interrogate 4096 sensors, albeit with a slight compromise in phase resolution. The number of interrogation sensors could be further increased by increasing the number of wavelength channels. We consider that this architecture, based purely on commercially available components, represents the best approach to support a distributed interferometric sensing array of more than 1000 sensors through one pair of interlink fibers.

\section{REFERENCES}

[1] P. J. Nash, G. A. Cranch, and D. J. Hill, "Large scale multiplexed fibre-optic arrays for geophysical applications," Industrial Sensing Systems, vol. 4202, pp. 55-65, 2000.

[2] G. A. Cranch and P. J. Nash, "Large-scale multiplexing of interferometric fiber-optic sensors using TDM and DWDM," Journal of Lightwave Technology, vol. 19, pp. 687-699, May 2001.

[3] C. W. Hodgson, M. J. F. Digonnet, and H. J. Shaw, "Large-scale interferometric fiber sensor arrays with multiple optical amplifiers," Optical Letters, vol. 22, pp. 1651-1653, 1997.

[4] C. W. Hodgson, J. L. Wagener, M. J. F. Digonnet, and H. J. Shaw, "Optimization of large-scale fiber sensor arrays incorporating multiple optical amplifiers.," Journal of Lightwave Technology, vol. 16, pp. 218-231, 1998.

[5] K. K. Clay and A. Dandrige, "Overview of high performance fibre-optic sensing," Journal of Physics D: Applied Physics, vol. 37, pp. 197-216, 2004.

[6] G. A. Cranch and P. J. Nash, "High multiplexing gain using TDM and WDM in interferometric sensor arrays," Fiber Optic Sensor Technology and Applications, vol. 3860, pp. 531-537, 1999.

[7] P. J. Nash, J. Latchem, G. Cranch, S. Motley, A. Bautista, C. Kirkendall, A. Dandridge, M. Henshaw, and J. Churchill, "Design, development and construction of fibre-optic bottom mounted array," 15th Optical Fiber Sensors Conference Technical Digest, pp. 333-336, 2002.

[8] A. D. Kersey, A. Dandridge, A. R. Davis, C. K. Kirdendall, M. J. Marrone, and D. G. Gross, "64-element time-division multiplexed interferometric sensor array with EDFA telemetry," Optical Fiber Communications, pp. 270-271, 1996.

[9] P. J. Nash and A. Strudley, "High efficiency TDM/WDM architectures for seismic reservior monitoring," 20th International Conference on Optical Fiber Sensors, vol. 7503, pp. T1-T4, 2009.

[10] G. A. Cranch, P. J. Nash, and C. K. Kirkendall, "Large-scale remotely interrogated arrays of fiber-optic interferometric sensors for underwater acoustic applications," Sensors Journal, vol. 3, pp. 19-30, 2003.

[11] C. W. Hodgson, M. J. F. Digonnet, and H. J. Shaw, "Large-scale interferometric fiber sensor arrays incorporating multiple optical switches," Optical Fiber Technology, vol. 4, pp. 316-327, 1998.

[12] J. L. Wagener, C. W. Hodgson, M. J. F. Digonnet, and H. J. Shaw, "Novel fiber sensor arrays using erbium-doped fiber amplifiers," Journal of Lightwave Technology, vol. 15, pp. 1681-1688, 1997.

[13] B. J. Vakoc, C. W. Hodgson, M. J. F. Digonnet, G. S. Kino, and H. J. Shaw, "Phase-sensitivity measurement of a 10 -sensor array with erbium-doped fiber amplifier telemetry," Optics Letters, vol. 23, pp. 1313-1315, 1998

[14] R. Slavik, Y. Liao, E. Austin, P. Petropoulos, and D. J. Richardson, "Full characterization and comparison of phase properties of narrow linewidth lasers operating in the C-band," 21st International Conference on Optical Fiber Sensors, vol. 7753, 2011

[15] Y. Liao, E. Austin, P. J. Nash, S. A. Kingsley, and D. J. Richardson, "Phase resolution characterization in fiber-optic sensor systems using amplifiers and TDM," unpublished.

[16] T. A. Berkoff and A. D. Kersey, "Signal-processing techniques for absolute displacement strain sensing using a fiber interferometer," Optics and Lasers in Engineering, vol. 16, pp. 153-161, 1992. 